\title{
Luces y sombras en los procesos de rehabilitación psicosocial de los enfermos psicóticos
}

RESUMEN: En este trabajo se han revisado los estudios sobre la eficacia de las medidas terapéuticas en rehabilitación psicosocial y se han analizado los recursos disponibles de las redes asistenciales rehabilitadoras.

Respecto a las medidas terapéuticas se intenta perfilar las más eficaces; y respecto a las redes de los dispositivos asistenciales, se focaliza la atención en la red rehabilitadora psicosocial española, llegando a la conclusión de que es aún insuficiente en número de dispositivos y en medios. Se finaliza haciendo propuestas para aumentar y mejorar la red asistencial rehabilitadora.

PALABRAS CLAVE: Rehabilitación, psicosocial, pacientes psicóticos, red asistencial.

\begin{abstract}
SUMMARY: Having review the existing studies on the efficiency of the therapeutic means at our disposal for psychosocial rehabilitacion, these are then analyzed with regard to the services available in our rehabilitation network.

Concerning the therapeutic measure, the authors try to iddentify these that would be most efficient, and with regard to the networking of rehabilitation services, we focus on the Spanish system. We find it insufficient in the number of service offered, as well as the means and resourdes. Concrete proposals are thereby formulated with a view to incrementing and bettering the psychosocial rehabilitation network.

KEY WORDS: Psychosocial,: rehabilitacion, psychotic patients, care services network.
\end{abstract}

\section{Introducción y objetivos}

La rehabilitación psicosocial de los pacientes psicóticos está experimentando un notable auge en nuestro país, como sucediera en otros países occidentales en las últimas décadas y como sin duda sucederá en otros países, cuyo desarrollo asistencial psiquiátrico vaya algo más retrasado.

Porque, ciertamente, la organización y desarrollo de la asistencia psiquiátrica y de salud mental en los diferentes países, comunidades autónomas y provincias suelen venir dados por la atención directa a los enfermos, en los ámbitos hospitalario y extrahospitalario, en primer lugar, y posteriormente van desarrollándose las estructuras rehabilitadoras.

También sucede que las estructuras rehabilitadoras van naciendo y creciendo de forma irregular, al amparo de planes más o menos completos o más o menos puntuales y gracias a las iniciativas creadoras de profesionales de la Salud Mental y con el apoyo - también desigual - de los Servicios Sociales. Estos suelen tener unas coberturas de Rev. Asoc. Esp. Neuropsiq., 2002, vol XXII, n. ${ }^{\circ}$ 84, pp. 155-163 
atención y unas prioridades, que no siempre coinciden con las necesidades de los enfermos psicóticos. En muchas ocasiones no son atendidas estas necesidades, precisamente por proceder los usuarios de la red de Salud Mental.

Los gestores de los Servicios Sociales, sin duda con la intención del mejor aprovechamiento de los recursos existentes, consideran que los enfermos mentales, al menos, están siendo tratados y atendidos en la red psiquiátrica y por lo tanto no es una prioridad, para ellos, la recuperación funcional psicosocial de los enfermos psicóticos.

Puede aplicarse este criterio para los equipos de Salud Mental, al menos en parte. Estando ocupados en el tratamiento y en el seguimiento de los pacientes, en su vertiente psicopatológica, para muchos de ellos no es prioritario que los pacientes sean incluidos en programas rehabilitadores, y que los procesos de rehabilitación psicosocial sean la continuidad lógica a los tratamientos psiquiátricos.

No obstante, una vez superadas las preocupaciones de los profesionales de la salud mental por la desinstitucionalización de los enfermos mentales, iniciada en los años 50-60, y en nuestro país en los 70, ha pasado a ocupar su interés el tema de la rehabilitación, que a partir de los años 80 se ha venido entendiendo como el intento de conseguir el mayor nivel de adaptación del paciente a la comunidad en que vive, buscando el modo de que su funcionamiento social sea lo más parecido a la normalidad. Sin duda, en este interés actual por la rehabilitación del enfermo mental ha jugado un papel capital la comprobación de que tanto las técnicas farmacológicas como las psicoterapéuticas no han permitido hasta el momento ir más allá en la mejoría del paciente (1), en cuanto a la normalización se refiere.

Existen distintos modelos de abordaje rehabilitador: unos que se apoyan en las estructuras y otros que lo hacen en el servicio, unos que defienden los centros de rehabilitación (centro de rehabilitación psicosocial, unidad de rehabilitación, centro ocupacional...), y otros el servicio de atención comunitaria (familia, medio social, centro de rehabilitación...).

Creemos que no está clara la concepción, y menos la puesta en práctica de medidas rehabilitadoras para el enfermo mental, y si, como hace unos años señalaba Sarraceno (2), la rehabilitación es "una práctica en espera de una teoría", nos ha parecido conveniente aportar nuestra visión sobre este aspecto tan importante del abordaje terapéutico del paciente crónico y del que tiende a la cronicidad.

Los objetivos que nos hemos propuesto en este estudio han sido los siguientes:

- Recoger información de la situación actual de la rehabilitación psiquiátrica en España, y de la eficacia a escala internacional de las intervenciones en rehabilitación psicosocial.

- Valorar las ventajas e inconvenientes de los diferentes recursos y modelos de organización de la rehabilitación psiquiátrica en España.

- Elaborar unas conclusiones sobre mejoras y alternativas a la situación actual de la rehabilitación psiquiátrica en España. 
INFORMES

\section{Metodología}

Se ha consultado la base de datos Medline de los últimos cinco años, en la búsqueda de revisiones sobre estudios de eficacia y efectividad de las medidas terapéuticas en rehabilitación psiquiátrica, así como otros estudios que han parecido significativos para los objetivos de este trabajo.

Se ha realizado una búsqueda directa de artículos o trabajos españoles en revistas o presentaciones a congresos y reuniones científicas, y en otros documentos.

Se han discutido los datos encontrados teniendo muy en cuenta la experiencia en Valladolid, donde trabajan los autores.

\section{Resultados y discusión}

Del material recogido, de los datos analizados y de nuestra propia experiencia de los últimos años, se desprenden las siguientes observaciones:

1. Podemos apreciar en nuestra actividad clínica diaria que un cambio en los síntomas no produce necesariamente una mejoría del funcionamiento general en un paciente esquizofrénico, por ello todas las definiciones sobre rehabilitación psicosocial hacen referencia a la adaptación, el funcionamiento social, la integración, etc.

Nos parece que en este marco adquiere un significado peculiar lo señalado por Bachrach (3) citando a Gittelman (4). Nos referimos a la sutil precisión de considerar la rehabilitación psicosocial como parte de la prevención secundaria en psiquiatría y no de la terciaria como parte del tratamiento en vez de ser algo que sigue al mismo.

2. En el ámbito internacional existe una gran variedad de abordajes y de coberturas de rehabilitación psicosocial, que van desde amplias redes rehabilitadoras con coberturas muy aceptables hasta descoberturas y lagunas que muestran notables deficiencias. Como también existe variedad de sistemas que van desde la inclusión de los dispositivos rehabilitadores en la propia red de Salud Mental, hasta el otro extremo de la inclusión de los dispositivos rehabilitadores en los Servicios Sociales, pasando por diversas formas de sistemas mixtos, en que los dispositivos rehabilitadores pertenecen a ambas redes e incluso a instancias independientes.

3. En el ámbito español predominan los sistemas mixtos, con un abanico de actividades diversas y unos dispositivos aún poco o nada coordinados e integrados en la mayoría de las Comunidades Autónomas. Los planes socio-sanitarios, todavía sin desarrollar plenamente, no han producido su esperado efecto de cobertura, ampliación e integración.

En relación con la rehabilitación psicosocial en España, nos hemos apoyado en el estudio realizado sobre su situación por el grupo de trabajo de la Asociación Española de Neuropsiquiatría (5), que aporta como datos más significativos los siguientes:

a) No existe uniformidad de criterios a la hora de definir los distintos dispositivos en rehabilitación y aparece una discordancia entre los datos oficiales y los facilitados por los profesionales sanitarios. Se observan servicios insuficientes y descoordinados con desarrollo muy desigual de unas comunidades a otras. 
b) Se recomienda establecer una atención prioritaria a la población susceptible de medidas de rehabilitación, previa su identificación, así como potenciar y organizar una completa red de recursos que esté coordinada. Se señala al mismo tiempo que al poner en marcha intervenciones psicosociales se haga siempre de acuerdo a la evidencia científica que exista al respecto.

c) Asimismo se apunta la importancia de establecer sistemas de evaluación de la eficacia de las intervenciones en rehabilitación, y de evaluación de los costes para garantizar su financiación.

La situación en nuestro país ha progresado desde el Informe del Defensor del Pueblo de 1991, pues entonces no aparecían datos de la existencia de talleres o empleos protegidos en casi ninguna comunidad española, mientras que en los datos del estudio del grupo de trabajo de la Asociación Española de Neuropsiquiatría se citan estructuras de este tipo en 9 de las 17 Comunidades Autónomas españolas. Algo semejante podría decirse de la existencia de centros de rehabilitación y alojamientos protegidos.

Hay que señalar que lo que en este estudio se entiende por alojamientos protegidos incluye pisos tutelados, pensiones y miniresidencias. Por empleo protegido se entienden talleres protegidos, empleo protegido, centro de formación laboral. Y en centros de rehabilitación se incluyen centros de día y unidades de rehabilitación y centros de rehabilitación psicosocial.

Lo destacable de los datos consultados es su gran variabilidad, oscilando, por ejemplo entre una tasa de 7 plazas en centros de rehabilitación x 100.000 habitantes de Murcia y 27 plazas en Castilla la Mancha y Extremadura.

En cuanto a alojamientos protegidos, la tasa oscila entre 1 Hogar x 100.000 habitantes en Extremadura y 12'6 en el País Vasco.

Y con relación a empleos protegidos, desde 1 plaza x 100.000 habitantes en Canarias a 21 en Andalucía, por poner solamente algún ejemplo.

En los años 80 las estimaciones que en EEUU se hacían en el Plan Nacional para Enfermos Mentales Crónicos (6) eran, de 400 a 700 pacientes crónicos no institucionalizados por 100.000 habitantes. Esta tasa es válida actualmente si tenemos en cuenta la prevalencia vida de la Esquizofrenia (por citar la psicosis más invalidante), que es del 1\%, y si, asimismo, tenemos en cuenta lo aceptado en la literatura (7) de que entre un $40 \%$ y un $60 \%$ de los esquizofrénicos ven su vida notablemente alterada como consecuencia de su enfermedad. Esto nos daría una tasa de crónicos necesitados de rehabilitación de entre 400 y 600 por 100.000 habitantes, lo que supondría que necesitarían claramente medidas de rehabilitación en España entre 160.000 y 240.000 pacientes, y en una provincia española media de unos 500.000 habitantes las necesitarían entre 2.000 y 3.000 pacientes.

4. Si pasamos a ocuparnos de la organización asistencial, en rehabilitación, hay que partir de que la rehabilitación - más que un conjunto de técnicas - (8) es una filosofía asistencial, por lo que la eficacia de los servicios dependerá más de cómo se conciben, y de su disponibilidad, que de las técnicas a aplicar (10), (11). 
Nos han parecido especialmente significativos los criterios que aporta José García (1997) para la organización básica de unos servicios de rehabilitación: organización comunitaria, diversificada, integral, aseguradora de continuidad de cuidados, a través de un equipo multidisciplinar, con intervención de las familias. Apoya el equipo de salud mental como elemento fundamental en la continuidad de cuidados en rehabilitación.

No debe olvidarse que la eficacia de una intervención orientada a la integración en la comunidad social no depende sólo de las habilidades que se adquieran, sino de en qué medida éstas se puedan hacer extensivas al medio habitual de vida del sujeto, y que con frecuencia el lugar donde se lleva a cabo el aprendizaje de estas habilidades es muy diferente de aquél en el que se va a desarrollar la vida del paciente (11), lo que no deja de ser paradójico. Tampoco hay que perder de vista que en la rehabilitación, así como en la cronificación psiquiátrica, hay que tener siempre en cuenta cuatro elementos: los pacientes, las familias, los profesionales y la sociedad (actitud, papel de las administraciones...)

Aunque las medidas en rehabilitación en los diversos países difieren según la tradición psiquiátrica, el sistema de protección social, el peso relativo del sector público y privado, etc., queremos añadir, a lo ya dicho hasta ahora, ocho elementos esenciales en la organización de la rehabilitación señalados por Bachrach (3).

a) Las intervenciones deben ser individualizadas. Es preciso adecuar los cuidados a cada paciente específico.

b) Tener siempre en cuenta el entorno del paciente, en el sentido de que sus capacidades deben adaptarse a la realidad del entorno o bien intentar adaptar el entorno a las posibilidades del paciente.

c) La rehabilitación debe estar orientada a los puntos fuertes del paciente; se debe trabajar con la parte sana de su yo.

d) El fin de la rehabilitación debe ser restablecer la confianza del individuo, siendo ése un elemento central en la rehabilitación.

e) La rehabilitación exige optimismo en las capacidades vocacionales del individuo enfermo, teniendo siempre en cuenta que el trabajo aporta muchas ventajas al paciente (recursos económicos que le facilitan autonomía, la posibilidad de ajustarse a un espacio a un tiempo, ampliar contactos con las demás personas, un papel en el reconocimiento social, y un motor para estar activo e implicado en algo).

f) La rehabilitación debe aportar una serie de aspectos lúdicos y sociales para la vida.

g) Exige que los pacientes estén activamente implicados en su propio cuidado, por tanto en el diseño de sus propios protocolos de rehabilitación.

h) La rehabilitación es un proceso continuo en el tiempo y en los diversos lugares, donde se encuentra el paciente con otros pacientes. La rehabilitación está intimamente ligada al concepto de continuidad de cuidados.

5. En el ámbito de la provincia de Valladolid se dan los siguientes hechos:

(a) Se confirma que el número de pacientes que se benefician de algún programa de rehabilitación psicosocial es muy pequeño, en comparación con el total de los pacientes psicóticos crónicos que lo necesitarían. 
Los pacientes que necesitarían algún tipo de rehabilitación psicosocial, en Valladolid, llegan a un número que se sitúa entre los 2000 y los 2300 según la estimación hecha a partir de los pacientes tratados en nuestra red asistencial psiquiátrica. El número de pacientes que realmente está recibiendo alguna atención rehabilitadora es de 387 (según la última recogida de datos efectuada). Por lo tanto, no llega al $20 \%$ de los pacientes que necesitarían rehabilitación psicosocial, los que realmente están en algún programa rehabilitador o en algún marco de actividad rehabilitadora.

(b) Los pacientes que están incluidos en algún programa rehabilitador, fuera de nuestros servicios, es relativamente pequeño, incluyendo los dispositivos de la red privada y los de la Asociación de Familiares de Enfermos Mentales, que en total no llegan a un centenar de pacientes, los que están en los dispositivos rehabilitadores mencionados.

$\mathrm{Si}$ a esto añadimos el hecho de que algunos de estos pacientes están en varios programas, simultáneamente - por ejemplo, en el Centro Ocupacional público y en actividades ludoterapéuticas y sociales del Centro de la Asociación de Familiares de Enfermos Mentales - se puede afirmar que apenas la quinta parte de los pacientes psicóticos crónicos está en algún programa rehabilitador y tiene seguimiento continuado.

(c) También se desprende de este análisis, que el campo más descubierto y abandonado ( y que requeriría mayor impulso) es el de la adquisición o recuperación de hábitos de trabajo y de promoción de empleo.

Solamente entre un $12 \%$ y un $14 \%$ de los pacientes de los Servicios Rehabilitadores de nuestra red entran en los programas ocupacionales de hábitos de trabajo y promoción al empleo directo o indirecto.

En términos globales, ésta es una pequeña proporción de pacientes, la de los que reciben asistencia y apoyo en rehabilitación laboral.

Si de todos los pacientes que necesitarían estructuras y programas rehabilitadores, menos de la quinta parte los reciben realmente, y de esta escasa quinta parte entran en programas de rehabilitación laboral solamente entre el $12 \%$ y el $14 \%$ de ellos, veremos que del total de pacientes psicóticos crónicos, de la provincia de Valladolid - que es nuestra área de competencia en salud mental - sólo reciben apoyo rehabilitador orientado hacia el trabajo entre un $2 \%$ y un $3 \%$ de los pacientes que, en principio, lo necesitarían. Si bien, somos conscientes de que no todos los pacientes y no todas las familias desean la recuperación de funciones orientadas al trabajo.

6. Muchos pacientes psicóticos crónicos no pasan del circuito asistencial al rehabilitador. Y otros muchos abandonan prematuramente el circuito rehabilitador, sin que haya una verdadera continuidad en el seguimiento. A nuestro entender, por los siguientes factores:

a) Por falta de coordinación entre los Servicios Asistenciales de Salud Mental y los Servicios Rehabilitadores.

b) Por falta de coordinación entre unos dispositivos rehabilitadores y otros.

c) Por falta de apoyo de las familias a los procesos rehabilitadores.

d) Por falta de apoyo suficientes de los Servicios Sociales. 
7. Como consecuencia de todos estos factores, los intentos rehabilitadores se quedan, en muchas ocasiones, en aspectos parciales de la rehabilitación, y muchos de los procesos rehabilitadores que se ofrecen a los pacientes se quedan en os aspectos socioculturales y lúdicos de la recuperación funcional y de la rehabilitación psicosocial.

8. Por último, en la revisión que hemos realizado sobre ensayos controlados de intervenciones psicosociales en la esquizofrenia, desde los años ochenta hasta hoy (12), así como las revisiones de la Cochrane Library (13-16), nos hemos encontrado con los datos siguientes:

a) Áreas específicas de interés clínico en las que son efectivas las siguientes técnicas:

Las intervenciones psicoeducativas, para aumentar la conciencia de enfermedad y el cumplimiento terapéutico, siempre que sean parte de un programa global de tratamiento de los pacientes psicóticos.

La terapia familiar y terapia comunitaria asertiva, en la prevención de recaídas psicóticas y reingresos hospitalarios. En el caso del tratamiento comunitario asertivo se ha comprobado que más no es igual a mejor y que para muchos pacientes un tratamiento clínico de alta calidad y una buena oferta de servicios resulta igualmente muy eficaz. En las poblaciones que muestran un índice de recaídas bajo, el aporte de una terapia de familia no constituye ventaja alguna.

El entrenamiento en habilidades sociales, para el aprendizaje de determinadas conductas sociales.

Los programas de ayuda al empleo, para la obtención de empleo competitivo alucinaciones.

La terapia cognitivo-conductual, para el control de ideas delirantes y

b) Se concluye de los estudios realizados que es necesario seguir investigando.

Para confirmar los resultados positivos que aportan los programas de ayuda al empleo y la terapia cognitivo-conductual en muestras más amplias y en condiciones más diversas de contexto e investigadores La Cochrane Library señala que los datos a favor o en contra no son concluyentes en cuanto a la rehabilitación cognitiva como tratamiento de la esquizofrenia

Para demostrar si los programas de terapia comunitaria asertiva suponen o no un ahorro de costes, aunque la Cochrane Library se inclina a favor de la reducción de costes hospitalarios con este tipo de intervención.

Con el fin de comprobar también la relación costo-efectividad de las terapias familiares psicoeducativas prolongadas.

Faltan estudios que orienten sobre la secuencia y combinación más idónea de los diferentes servicios ofertados.

La Cochrane Library indica que se necesitan más estudios para poder precisar la utilidad del entrenamiento en habilidades sociales.

c) Se recomienda en el ámbito clínico:

Terapia familiar psicoeducativa continuada para pacientes que sufren recaídas frecuentes y viven con sus familias. 
Programas de terapia comunitaria asertiva para los usuarios habituales de los servicios, sobre todo si la familia no se implica en el tratamiento.

Entrenamiento en habilidades sociales dado que una vez controlado el proceso psicótico no existen pruebas de que los fármacos ofrezcan ventajas adicionales al paciente para su capacidad de desenvolverse adecuadamente en la sociedad.

Siempre que sea posible, ofrecer un programa de ayuda al empleo.

\section{Conclusiones}

A nuestro entender, es imprescindible intentar cubrir las lagunas existentes en rehabilitación psicosocial, de manera que lleguen a satisfacerse las muchas necesidades existentes y que aún no tienen la respuesta adecuada. Si queremos conseguir dar una respuesta más amplia y más realista, hay que actuar decididamente en los siguientes aspectos:

1.- Influir en los poderes públicos y asesorarles para emprender una clara y enérgica política de salud mental, que incluya una sustancial aportación de medios para la creación de nuevas estructuras rehabilitadoras que, por otra parte, siempre serán menos costosas que las hospitalarias y que las asistenciales, en general.

2.- Hacer campañas de oferta de Servicios de Rehabilitación Psicosocial dirigidas a las grandes bolsas de población de enfermos psíquicos crónicos, o en vías de cronificación, que están sin atención rehabilitadora.

3.- Promover programas informativos de actuación sobre la pasividad y el desánimo de los psicóticos crónicos y de sus familiares, para estimularlas a entrar en los circuitos rehabilitadores y a continuar en ellos.

4.- Hacer un esfuerzo de coordinación entre los Servicios de Salud Mental, los Servicios Sociales y los Servicios de Rehabilitación Psicosocial para garantizar el aprovechamiento de recursos, el modo de derivación de los pacientes y asegurar la imprescindible continuidad.

5.- Potenciar la permeabilidad y la cooperación entre todos los dispositivos Rehabilitadores, para aumentar su capacidad y eficacia.

6.- Desarrollar dispositivos específicos de rehabilitación psicosocial que vayan más en profundidad en las técnicas rehabilitadoras y que estén en comunicación funcional con los Centros de Salud Mental y Servicios Sociales.

7.- Intentar proponer (desarrollar) una teoría de la práctica rehabilitadora respondiendo a la idea de Sarraceno de que la rehabilitación es una práctica sin teoría ofreciendo un substrato básico de filosofía asistencial-rehabilitadora. 
INFORMES

\section{BIBLIOGRAFÍA}

1 Sánchez, AE., "Consideraciones en torno a la rehabilitación", Psiquiatría Pública, 1992, 4, pp. 130-134.

2 Sarraceno, B., "Entrevista con Benedetto Sarraceno", Boletín de la AMRP, 1995, nº 3.

3 Bachrach, LL., "Psychosocial Rehabilitation and Psychiatry in the Care of Long-Term Patientes", Am. J. Psychiatry, 1992, 149, pp. 1455-1463.

4 Gittelman, M. "Recents developments in European rehabilitation programs for mental patients". In Bellak, L.; Barten HH., (ed.) Progress in Community Mental Health, New York, Burner/Mazel, 1975.

5 Gisbert, C.et al. "Rehabilitación psicosocial del trastorno mental sereno. Situación actual y recomendaciones". Cuadernos técnicos. 6 Madrid. AEN 2002

6 Szymanski, HV.; Schulberg, HC.; Salter, V.; Gutterman, N., "Estimating the Local prevalence of Persons Needing Community Support Programs", Hosp. Communituy Psychiatry, 1982, 33, pp. 370-373.

7 Kaplan, HI.; Sadock, BJ.; Greeb, JA., Sinopsis de Psiquiatría. (7ª ed), 1996. pp. 495-496.

8 Guinea, R., "Rehabilitación psicosocial: una teoría, una práctica, una ética", Rev Asoc. Esp. Neuropsiq., 1997, 65, pp. 151-164.

9 Shepherd, G., "Avances recientes en la rehabilitación psiquiátrica”, En Aldaz, JA.; Vázquez, C., (comps.), Esquizofrenia: fundamentos psicológicos y psiquiátricos de la rehabilitación. Madrid, Siglo XXI, 1996, pp. 1-22.

10 García, J.; de las Heras, B., Peñuelas, E. (ed.), Rehabilitación psicosocial: conceptos y estrategias. Oviedo, KRK, 1997.

11 Vázquez, C.; Muñoz, N.; Muñiz, E.; López, B.; Hernangómez, L.; Díez M., "La evaluación de resultados en el tratamiento y rehabilitación de trastornos mentales crónicos", Rev. Asoc. Esp. Neuropsiq., 2000, 20, pp. 207-228

12 Penn, DL.; Mueser, KT., "Research update on the psichosocial reatment of schizophrenia", Am. J..Psychiatry. 1996, 153, pp. 607-617

$13 \mathrm{http}: / /$ cochrane.org/cochrane/revabstr/ab003080.htm

$14 \mathrm{http} / / /$ cochrane.org/cochrane/revabstr/ab001089.htm

15 http://cochrane.org/cochrane/revabstr/ab000968.htm

16 http://cochrane.org/cochrane/revabstr/ab000381.htm

\section{*Psiquiatra}

Jefe Servicios Rehabilitadores en la Comunidad

(Servicios de Salud Mental de Valladolid)

**Psiquiatra

Jefe de Servicios Extrahospitalarios

(Servicios de Salud Mental de Valladolid)

Correspondencia. Fernando Leal Herrero. Centro Asistencial Dr. Villacián

C/ Orión, 2. 47014. Valladolid.

Recibido: 12-07-02 